\title{
The Effect of Two Different Sperm Cryopreservation Methods on The Integrity of Its DNA Content (a Comparative Study)
}

Mohammed M Farid Rayea ${ }^{1}$, Medhat Kamel Amer ${ }^{1}$, Ahmed Abdelhady Mousa ${ }^{2}$, Mohammed Gamal Farghaly ${ }^{1 *}$

${ }^{1}$ Department of Andrology, Sexology and STDs, Faculty of Medicine, Cairo University

${ }^{2}$ Department of Andrology, Sexology and STDs, Faculty of Medicine,Bani Suef University

*Corresponding author: Mohammed Gamal Farghaly, Mobile: (+20) 01003656609,

E-Mail: drmohamedgama193@yahoo.co

\begin{abstract}
Background: Sperm cryopreservation is currently the only clinically available technique for preservation of male gametes for subsequent use in assisted reproduction technologies.

Objective: The objective of this study was to determine the impact of semen cryopreservation on human sperm DNA.

Patients and methods: The study sample consists of 20 male patients seeking seminal fluid analysis at the andrology laboratory of a specialized IVF center (ADAM International Hospital for Fertility and Sterility, Giza, Egypt), and accepting to sign a consent of approval to participate in the study, with the diagnoses of normozoospermia, oligozoospermia (either isolated or combined with asthenozoospermia or teratozoospermia).

Results: There is no statistical difference between Pre-freezing and vitrification regarding DNA fragmentation, $P>0.05$. No statistical difference between Pre-freezing and slow frozen regarding DNA fragmentation, $P>0.05$. Finally, there is no significant statistical difference between vitrification and slow frozen regarding DNA fragmentation, $\mathrm{P}>0.05$.

Conclusion: The sperm DNA fragmentation index was not affected during cryopreservation under the various methods of storage tested. Clinicians and investigators should take this information into consideration when using cryopreserved sperm for assisted reproduction.
\end{abstract}

Keywords: Sperm cryopreservation, DNA content

\section{INTRODUCTION}

During freezing, the formation of ice in the aqueous medium in which the spermatozoa are suspended increases the concentration of the solutes in the extracellular environment, which will lead to the dehydration of cell. This process is necessary to protect the cells from the formation of intracellular ice. However, if the extent of dehydration is too severe, and the cell volume reaches approximately $40 \%$ of its original size, it could lead to irreversible sperm damage. Then, during thawing, the melting of extracellular ice leads to lower extracellular solutes concentration than the intracellular milieu in spermatozoa. Water then enters back inside the spermatozoa and restores its volume. However, there is a risk of formation of intracellular ice crystals during this phase, which could lead to irreversible cell damage. Minimizing the formation of intracellular ice crystals is highly important for the survival of spermatozoa after cryopreservation ${ }^{(\mathbf{1})}$.

Extenders are used to preserve the survival of spermatozoa after freeze- thawing. The composition of these media is variable, but they usually contain a buffer to preserve extracellular $\mathrm{pH}$ and osmolarity; sugars as a source of energy for the spermatozoa; antibiotics; and most importantly, a cryoprotectant such as glycerol. Glycerol can pass through sperm membrane and decrease the freezing point of water, thus decreasing the risk of intracellular ice formation during freezing ${ }^{(2)}$.

The sperm DNA damage analysis has become a complementary biomarker in determining male infertility ${ }^{(3)}$. Sperm DNA fragmentation (SDF) has been developed as a marker of sperm DNA quality, and many studies have shown an increase in SDF in infertile patients compared with fertile donors, and have established clinical threshold values for infertility using different techniques ${ }^{(4,5)}$.

With the growing interest in reproductive medicine on the impact of sperm DNA integrity on reproductive outcomes, especially with assisted reproduction, it is important to evaluate whether cryopreservation could affect sperm DNA integrity. Different assays are used to assess the integrity of sperm chromatin and sperm DNA. Each assay measures different parameters such as sperm DNA strand breaks ${ }^{(5,6)}$, sperm chromatin structure (SCSA ${ }^{\circledR}$ assay), or sperm DNA compaction (CMA3 assay) ${ }^{(6,7)}$.

Gamete cryopreservation is widely used for a variety of purposes, such as fertility preservation before chemotherapy treatment, donor sperm cryopreservation $^{(\mathbf{8})}$. Because of that, it is important to understand the effects of cryopreservation to preserve the better quality of the thawed sample. It has been shown that cryopreservation reduces 
sperm motility and sperm vitality ${ }^{(9)}$. Some studies focused on the effect of cryopreservation on sperm DNA damage, showing that the main effector of DNA damage during the process of freezing and thawing a semen sample are the reactive oxygen species ${ }^{\left({ }^{10}\right)}$. However, the effect of cryopreservation on sperm DNA integrity remains controversial with some reports showing an effect ${ }^{(\mathbf{1 1})}$, whereas others report none ${ }^{(\mathbf{1 2})}$. These controversial data may be resolved by controlling for additional factors that affect sperm DNA integrity during freeze/thawing, such as the previous state of the sample ${ }^{(\mathbf{1 3})}$, the technique used for cryopreservation or the cryoprotectant applied ${ }^{(\mathbf{8})}$.

\section{AIM OF THE WORK}

The objective of this study was to determine the impact of semen cryopreservation on human sperm DNA.

\section{PATIENTS AND METHODS}

The study sample consists of 20 male patients seeking seminal fluid analysis at the andrology laboratory of a specialized IVF center (ADAM International Hospital for Fertility and Sterility, Giza, Egypt), and accepting to sign a consent of approval to participate in the study, with the diagnoses of normozoospermia, oligozoospermia (either isolated or combined with asthenozoospermia or teratozoospermia).

\section{Ethical approval}

The study was approved by the Ethics Board of Cairo University and an informed written consent was taken from each participant in the study.

Inclusion criteria: Semen analysis showing at least 1 motile sperm/drop after centrifugation, whatever the sperm count and morphology.

Exclusion criteria: Patients with immotile spermatozoa or pyospermia.

Semen samples were collected, and then semen analysis was done according to the WHO ${ }^{(14)}$.

Samples were classified into two groups:

- Group A: samples with the diagnosis of normozoospermia, mild or moderate oligozoospermia. (Spermatozoa count more than $5 \times 10^{6} / \mathrm{ml}$ ).

- Group B: samples with the diagnosis of severe oligozoospermia either isolated or combined with asthenozoospermia or teratozoospermia (count of or less than $5 \times 10^{6} / \mathrm{ml}$ down to at least 1 motile sperm after centrifugation).
- Sperm chromatin dispersion: Sperm chromatin dispersion was performed before and as soon as possible after thawing of slow frozen or vitrified samples.

\section{Procedure details}

Aliquots of raw semen and of the different isolate gradient fractions in mHTF medium will be analyzed directly after thawing. Samples will be thawed at room temperature and diluted in MHTF medium to obtain sperm concentrations that range between 5 and 10 million $/ \mathrm{mL}$. The halo kit (Halosperm, Spain) will be used in the test as follows:

The suspensions will be mixed with $1 \%$ lowmelting point aqueous agarose (to obtain a $0.7 \%$ final agarose concentration) at $37.8^{\circ} \mathrm{C}$. Aliquots of 50 micro Liters of the mixture will be pipette onto a glass slide precoated with $0.65 \%$ standard agarose dried at $80^{\circ} \mathrm{C}$, covered with a coverslip $(22$ by $22 \mathrm{~mm}$ ), and will be left to solidify at $4{ }^{\circ} \mathrm{C}$ for 4 minutes. As in the halo test or the comet assay, the agarose matrix allows for work with unfixed sperm on a slide in a suspension like environment. Cover slips will be carefully removed, and slides will be immediately immersed horizontally in a tray with freshly prepared acid denaturation solution $(0.08 \mathrm{~N}$ $\mathrm{HCl}$ ) for 7 minutes at $22{ }^{\circ} \mathrm{C}$ in the dark to generate restricted single-stranded DNA (ssDNA) motifs from DNA breaks. The denaturation will be then stopped, and proteins will be removed by a transfer of the slides to a tray with neutralizing and lysing solution (0.4 M Tris, 0.8 M DTT, $1 \%$ SDS, and 50 mM EDTA, pH 7.5, 0.4 M Tris, $2 \mathrm{M} \mathrm{NaCl}$, and $1 \%$ SDS, $\mathrm{pH}$ 7.5) for 25 minutes at room temperature. Slides will be thoroughly washed in distilled water 2 minutes, dehydrated in sequential $70 \%, 90 \%$, and $100 \%$ ethanol baths (2 minutes each), and air dried. Cells will be stained with the Diff-Quik reagent (Baxter Healthcare Corporation Inc, McGaw, Ill) for brightfield microscopy.

\section{Sample Preparation}

Samples were prepared (either by swim up or wash and centrifugation) before further assessment and cryopreservation by wash and centrifugation or by swim up, according to seminal fluid assessment; Normal, mild and moderate male factor sperms were prepared by swim up while severe oligozoospermia and severe male factor were prepared by wash and centrifugation ${ }^{(13)}$, (Modified Human tubal fluid $+1 \%$ Human Serum Albumin (mHTF - HAS), pure sperm wash $^{\circledR}$ Nidacon, Sweden). Quinn et al. ${ }^{(15)}$ recommendations were the basic medium used in sperm preparation.

The diluted suspension of spermatozoa was again diluted (1:1) with $0.5 \mathrm{M}$ sucrose solution $(0.25 \mathrm{M}$ 
end concentration). The $0.5 \mathrm{M}$ sucrose in bidistillate water then filtered with $0.22 \mathrm{~mm}$ filter then was frozen until use. After dilution, aliquots were maintained at $37.8^{\circ} \mathrm{C}$ for $5 \mathrm{~min}$ before the cooling procedure takes place. Then after proper mixing, each sample was split into 3 aliquots: Aliquot 1: control group (fresh ejaculate), Aliquot 2: spermatozoa cryopreserved by slow conventional freezing with glycerol-containing medium, Aliquot 3: spermatozoa vitrified in 0.3 $\mathrm{ml}$ insemination "French" straws in culture with $0.25 \mathrm{M}$ sucrose containing medium.

\section{Spermatozoa Cryopreservation:}

1. Spermatozoa Vitrification Technology of aseptic cryoprotectant-free vitrification of human spermatozoa in large volumes; the technology includes - Cryoprotective medium with only non-permeable cryoprotective agents (0.25 $\mathrm{M}$ sucrose in end concentration). As basal medium was (Modified Human tubal fluid medium $+1 \%$ Human Serum Albumin, Puresperm wash ${ }^{\circledR}$ Nidacon, Sweden). Using of $0.3 \mathrm{ml}$ plastic straws with subsequent sealing from both side before cooling in liquid nitrogen. The warming up of spermatozoa is achieved by immersing straw with vitrified spermatozoa into warmed water bath at $42^{\circ} \mathrm{C}$.

The packaging of spermatozoa for aseptic vitrification was performed in the following way: Spermatozoa suspensions were cooled in 0.3 $\mathrm{ml}$ plastic (CBS) straws (CryoBio System, Paris, France), The straw was labeled with asterisk $(1 \mathrm{~cm}$ from the inner end of cotton-polyvinyl plunge, the straw was filled up to asterisk with $0.3 \mathrm{ml}$ of spermatozoa suspension by aspiration. Then the filled straw was expelled from the tube while aspiration of air continued. Subsequently, when the suspension reached the polyvinyl plunge, the polymerization of polyvinyl initiated due to humidification. After aspiration was completed, and the top end of straw was sealed by polymerized polyvinyl, straw was heat-sealed at both sides by thermo-hermetic sealing. The straws were immersed into liquid nitrogen in horizontal position (approximately for 8 seconds) and were stored there at least for 24 hours before use. The warming up of spermatozoa was achieved by immersing straw with vitrified spermatozoa into water bath at $42^{\circ} \mathrm{C}$ and dangling it gently in water for 20 seconds. After warming, the residual fluid was removed from the straw with paper towel, and straw disinfected with $70 \%$ ethanol. The heatsealed part of straw (opposite to the cotton polyvinyl plunge) was cut off with sterile scissors, and the aspirator was connected with the straw. A low differential negative pressure was applied by aspiration. That ensures that after subsequent cutting of the cotton-polyvinyl plunge fluid won't be leaking out. Finally, the suspension was expelled from the straw for immediate evaluation of sperm quality.

\section{Conventional Spermatozoa Freezing}

The manual method of conventional freezing was performed by decreasing the temperature of the semen after adding a Glycerol cryoprotectant (Sperm Freeze Solution, Fertipro) (kept at $4-6{ }^{\circ} \mathrm{C}$ and placed at $37^{\circ} \mathrm{C}$ for $10-15 \mathrm{~min}$ before being ready to use), the cryoprotectants was added in an equal volume to semen in a dropwise manner, gently mixed at room temperature, in a stepwise manner and after that loading the specimen in 0.3 ml CBS straws (CryoBio System, Paris, France) and slowly cooling the specimen then plunging the samples into liquid nitrogen. The initial cooling rate of the specimen from room temperature to $5^{\circ} \mathrm{C}$ was $0.5-1^{\circ} \mathrm{C} / \mathrm{min}$. The sample was then frozen from $5^{\circ} \mathrm{C}$ to $-80^{\circ} \mathrm{C}$ at a rate of $1-10^{\circ} \mathrm{C} / \mathrm{min}$. The specimen was then plunged into liquid nitrogen at $-196^{\circ} \mathrm{C}$. To thaw the samples, the straws were taken from the liquid nitrogen, held in air for 30 seconds, immersed into $37^{\circ} \mathrm{C}$ water bath in the horizontal position and held in this bath for 20 seconds until the ice melted. After thawing, $10 \mathrm{~mL}$ of basic $\mathrm{m}$ HTF-HAS medium was added to the thawed sample and centrifuged for 5 minutes at $340 \mathrm{~g}$. The supernatant was removed and the pellet resuspended with the same basic medium in order to obtain a final concentration of $0.5 \times 10^{6} / \mathrm{ml}$.

\section{Statistical analysis}

Recorded data were analyzed using the statistical package for social sciences, version 20.0 (SPSS Inc., Chicago, Illinois, USA). Quantitative data were expressed as mean \pm standard deviation (SD). Qualitative data were expressed as frequency and percentage.

\section{The following tests were done:}

- Independent-samples t-test of significance was used when comparing between two means.

- Chi-square $\left(\mathrm{x}^{2}\right)$ test of significance was used in order to compare proportions between two qualitative parameters.

- The confidence interval was set to $95 \%$ and the margin of error accepted was set to 5\%. The pvalue was considered significant as the following:

- Probability (P-value)

- P-value <0.05 was considered significant.

- P-value <0.001 was considered as highly significant.

- P-value >0.05 was considered insignificant. 


\section{RESULTS}

Comparison between group A regarding DNA fragmentation (No=10)

Table (1): Comparison between group A regarding DNA fragmentation

\begin{tabular}{|c|c|c|}
\hline Aliquot & Mean \pm SD & P-value \\
\hline $\begin{array}{c}\text { Pre-freezing } \\
\text { VS } \\
\text { Vitrification }\end{array}$ & $\begin{array}{c}9.0 \pm 7.2 \\
\mathrm{VS} \\
15.8 \pm 13.4\end{array}$ & 0.176 \\
\hline $\begin{array}{c}\text { Pre-freezing } \\
\text { VS } \\
\text { Frozen }\end{array}$ & $\begin{array}{c}9.0 \pm 7.2 \\
\text { VS } \\
12.6 \pm 11.3\end{array}$ & 0.408 \\
\hline $\begin{array}{c}\text { Vitrification } \\
\text { VS } \\
\text { Frozen }\end{array}$ & $\begin{array}{c}15.8 \pm 13.4 \\
\text { VS } \\
12.6 \pm 11.3\end{array}$ & 0.574 \\
\hline
\end{tabular}

\#: Paired t test

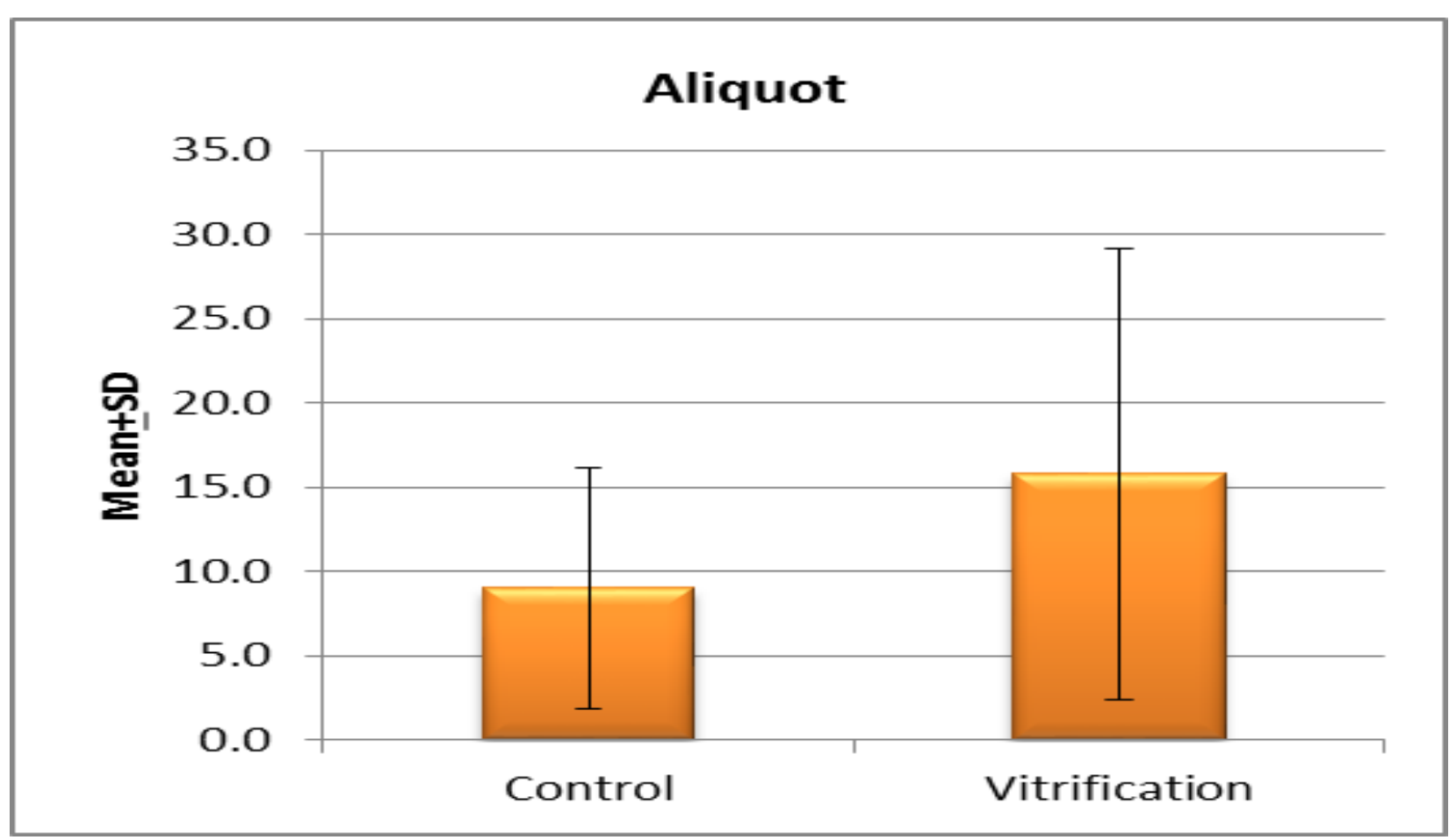

Fig. (1): Comparison between Pre-freezing and Vitrification regarding DNA fragmentation. 


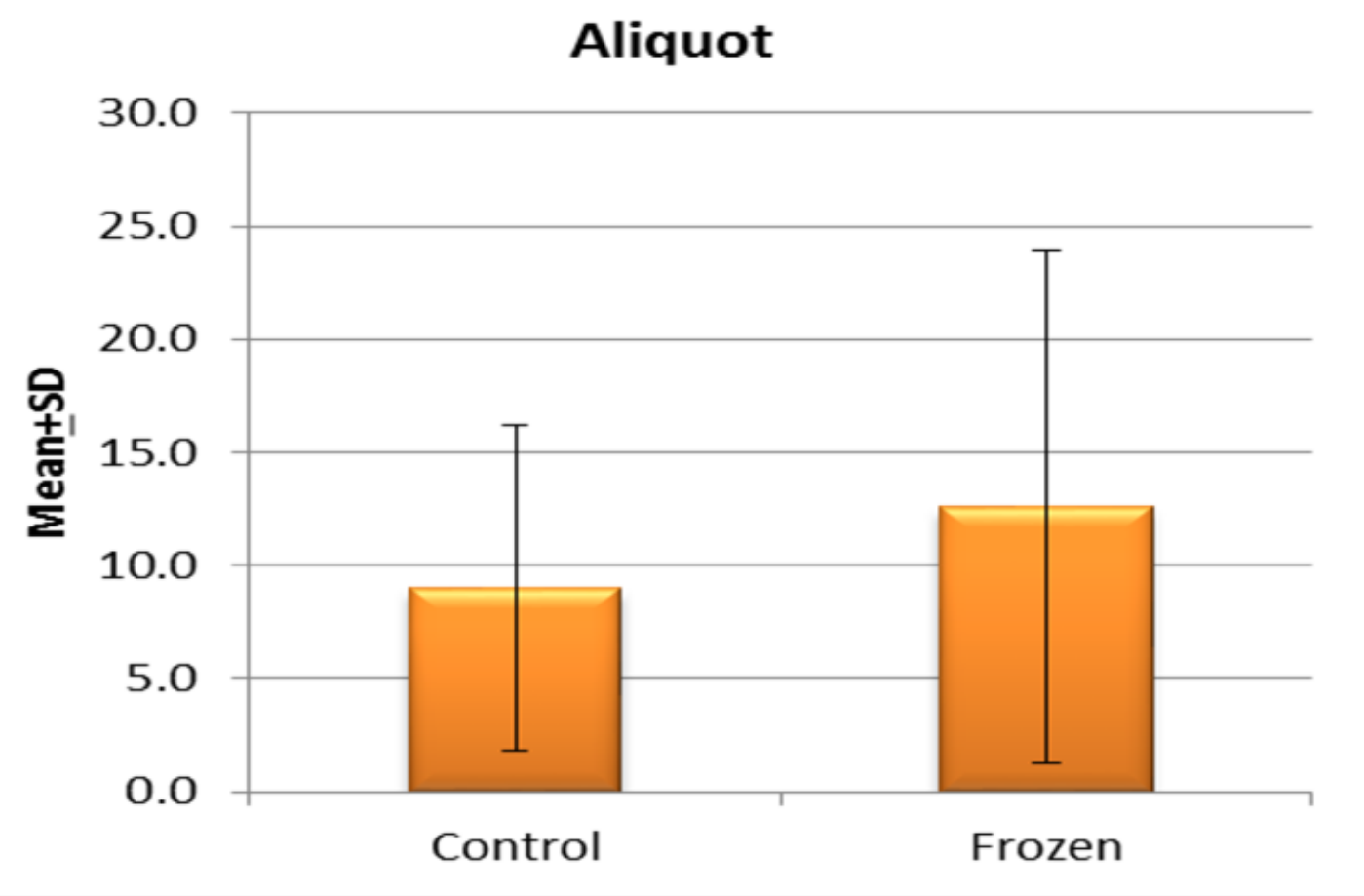

Fig. (2): Comparison between Pre-freezing and slow Frozen regarding DNA fragmentation.

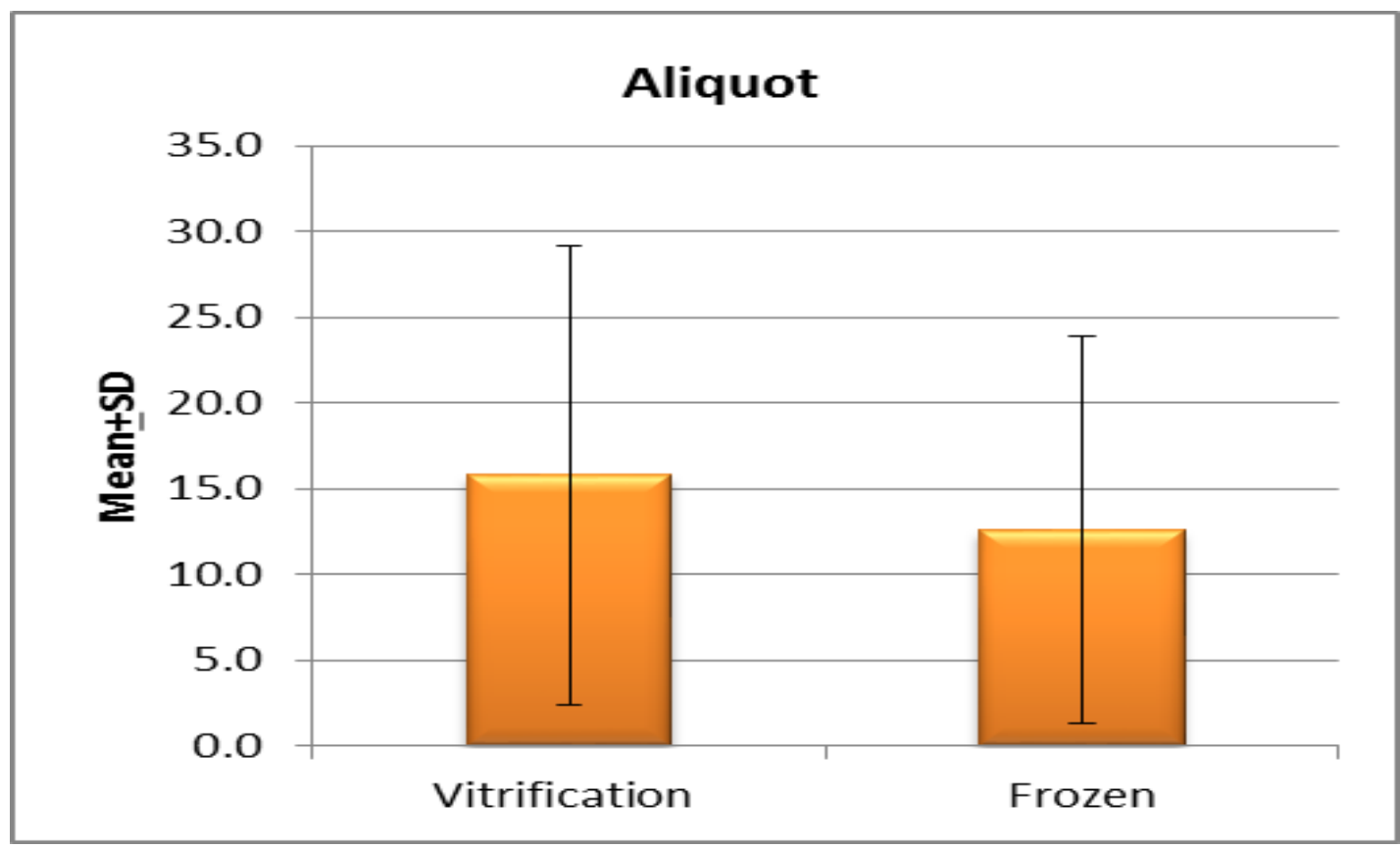

Fig. (3): Comparison between Vitrification and slow Frozen regarding DNA fragmentation.

Table 1 and figures 1,2,3 illustrate the mean levels of group A regarding DNA fragmentation, the highest mean is vitrification followed by slow Frozen and lowest mean is at Pre-freezing group (15.8, 12.6, and 9.0 respectively),There is no statistical difference between Pre-freezing and vitrification regarding DNA fragmentation, $\mathrm{P}>0.05$. No statistical difference between Pre-freezing and slow frozen regarding DNA fragmentation, $P>0.05$. Finally, there is no significant statistical difference between vitrification and slow frozen regarding DNA fragmentation, $\mathrm{P}>0.05$. 


\section{DISCUSSION}

Cryopreservation is the collection, freezing, and long term storage of sperm, and is a highly effective method of protecting male fertility. Cryopreservation of semen has been widely used as a vital method for fertility preservation of male patients before undergoing chemotherapy, radiotherapy, and/or surgery that may lead to testicular failure or ejaculatory dysfunction (16). Freezing of sperm before initiation of treatment provides patients with "fertility insurance " and may allow them to father their own children using intrauterine insemination (IUI), conventional invitro fertilization (IVF) or intracytoplasmic sperm injection (ICSI) ${ }^{(17)}$.

There are two main conventional freezing techniques used in sperm cryopreservation: slow freezing and rapid freezing. The slow freezing technique proposed by Behrman and Sawada ${ }^{(18)}$ and rapid freezing was first proposed by Sherman (19).

According to the scientific achievements in cryobiology at present; the vitrification belongs to perspective technologies ${ }^{(20)}$. Because; a) The method is cheap and quick, b) Due to lowering the temperature of solution's glass transition the permeable cryoprotectants prevents the actual freezing of solution and allows maintaining its same flexibility in a glassy phase, and c) Vitrification without permeable cryoprotectants allows avoiding the cryoprotectants toxicity and osmotic stress; the damage of plasmatic and mitochondrial membrane during equilibration with cryoprotectants; and protects them against lipid peroxidation and formation of reactive oxygen species and DNA damage.

Vitrification is an alternative method that can also be applied to achieve the same purpose and does not use the special extenders. This method is based on the rapid cooling of the cells by immersion into liquid nitrogen, and, thereby, is the key factor reducing the chance of the formation of big ice crystals ${ }^{(20)}$.

In contrast to the programmable ("slow") conventional freezing, vitrification has series of technological advantages useful for the practice: it renders the use of permeable cryoprotectants superfluous and, in addition, is much faster, simpler in application and more cost-effective than conventional freezing ${ }^{(20)}$.

The objective of this study was to determine the impact of semen cryopreservation on human sperm DNA.

The study sample consists of 20 male patients seeking seminal fluid analysis at the andrology laboratory of a specialized IVF center (ADAM International Hospital for Fertility and Sterility,
Giza, Egypt), and accepting to sign a consent of approval to participate in the study, with the diagnoses of normozoospermia, oligozoospermia (either isolated or combined with asthenozoospermia or teratozoospermia).

In our study, Vitality of vitrified spermatozoa showed significant statistically lower levels as compared to conventional slow freezing. It was shown in different groups that vitality of vitrified spermatozoa decreases significantly in comparison with slow freezing as we go through the different times (post thawing and 1-hour and 24-hours). This is contrary to results of another study found that higher rates of CMI were achieved in vitrified sperm as compared to slow conventional freezing. However, as compared to nontreated controls (fresh spermatozoa) both cryopreservation procedures had a significant impact on viability ${ }^{(21)}$.

Some studies showed no statistical differences in parameters such as viability, recovery rate or percentage of morphologically normal spermatozoa with undamaged DNA between vitrified and conventionally frozen cells (22).

In our study, DNA fragmentation of vitrified spermatozoa showed higher levels as compared to conventional slow freezing but there is no significant statistical difference between vitrification and conventional slow freezing in DNA fragmentation.

Encouraged by the findings of the German group, the Canadians (as Moskovtsev) have also looked at possibilities to utilize vitrification in their laboratory. They have compared sperm motility, kinetics and DNA damage between semen samples cryopreserved by standard vapor freezing verses vitrification protocols ${ }^{(23)}$.

Their results indicate that sperm motility was significantly reduced for both types of frozen/thawed samples. Mean motility of vitrified samples was decreased when compared to samples prior to freezing, which was almost two-fold higher compared to motility of samples frozen by standard slow vapor protocol, and also was decreased when compared to samples prior to freezing.

Sperm kinematics such as VCL, VSL, and LIN were not significantly different between the two types of cryopreservation protocols without taking into account CASA- paradox.

However, when MKP were calculated, it was revealed that indeed vitrified samples had superior recovery of sperm kinematic parameters in comparison to slow freezing.

They found statistically significant increase in sperm DNA damage after both methods of sperm freezing. However, the increase in DNA 
damage was minimal and to a degree probably irrelevant to clinical concerns. No significant differences were observed in sperm DNA damage between slow freezing and vitrification.

They can confirm from previous reports that human spermatozoa can be successfully vitrified without the use of potentially toxic cryoprotectants. The vitrification protocol showed significantly better results in preserving motility rates of spermatozoa when compared to slow vapor freezing. No significant differences were observed in post thaw sperm DNA damage in comparison to the standard slow freezing method ${ }^{(23)}$.

\section{CONCLUSION}

The sperm DNA fragmentation index was not affected during cryopreservation under the various methods of storage tested. Clinicians and investigators should take this information into consideration when using cryopreserved sperm for assisted reproduction.

\section{REFERENCES}

1. Paoli D, Lombardo F, Lenzi A et al. (2014): Sperm cryopreservation: effects on chromatin structure. In: Genetic Damage in Human Spermatozoa (eds. E Baldi \& M Muratori), Springer, New York, Pp. 137-150.

2. Holt WV (2000): Basic aspects of frozen storage of semen. Anim Reprod Sci., 62: 3-22.

3. Templado C, Vidal F, Estop A (2011): Aneuploidy in human spermatozoa. Cytogenet Genome Res., 133: 91-99.

4. Simon L, Lutton D, McManus J et al. (2011): Sperm DNA damage measured by the alkaline Comet assay as an independent predictor of male infertility and in vitro fertilization success. Fertil Steril., 95: 652-657.

5. Ribas-Maynou J, Fernandez-Encinas A, GarciaPeiro A et al. (2014): Human semen cryopreservation: a sperm DNA fragmentation study with alkaline and neutral Comet assay. Andrology, 2: 83-87.

6. Rahiminia T, Hosseini A, Anvari $M$ et al. (2017): Modern human sperm freezing: effect on DNA, chromatin and acrosome integrity. Taiwan J Obstet Gynecol., 56: 472-476.

7. Delbes G, Hales BF, Robaire B (2010): Toxicants and human sperm chromatin integrity. Mol Hum Reprod., 16: $14-22$.

8. Di Santo M, Tarozzi N, Nadalini M et al. (2012): Human sperm cryopreservation: update on techniques, effect on DNA integrity, and implications for ART. Adv Urol., 2012: 854-7.

9. Satirapod C, Treetampinich C, Weerakiet S et al. (2012): Comparison of cryopreserved human sperm from solid surface vitrification and standard vapor freezing method: on motility, morphology, vitality and DNA integrity. Andrologia, 44: 786-790.

10.Said TM, Gaglani A, Agarwal A (2010): Implication of apoptosis in sperm cryoinjury. Reprod Biomed Online, 21: 456-462.

11.Zribi N, Feki Chakroun N, El Euch H et al. (2010): Effects of cryopreservation on human sperm deoxyribonucleic acid integrity. Fertil Steril., 93:159166.

12. Isachenko E, Isachenko V, Katkov II et al. (2004): DNA integrity and motility of human spermatozoa after standard slow freezing versus cryoprotectant-free vitrification. Hum Reprod., 19: 932-939.

13. Ahmad L, Jalali S, Shami SA et al. (2010): Effects of cryopreservation on sperm DNA integrity in normospermic and four categories of infertile males. Syst Biol Reprod Med., 56:74-7.

14. WHO (2010): Laboratory manual for the examination and processing of human semen, $5^{\text {th }}$ ed. Geneva, World Health Organization. https://apps.who.int/ iris/handle/10665/44261

15. Quinn P, Warnes GM, Kerin JF et al. (1985): Culture factors affecting the success rate of in vitro fertilization and embryo transfer. Ann NY Acad Sci., 442:195-204.

16. Tournaye H, Devroey P, Liu J et al. (1994): Microsurgical epididymal sperm aspiration and intracytoplasmic sperm injection: a new effective approach to infertility as a result of congenital absence of the vas deferens. Fertil Steril., 61:1045-1051.

17.Li Z, Lin Q, Liu R et al. (2010): Protective effects of ascorbate / and catalase on human spermatozoa during cryopreservation. J Androl., 31 :5-8.

18. Behrman SJ, Sawada Y (1966): Heterologous and homologous inseminations with human semen frozen and stored in a liquid-nitrogen refrigerator. Fertility and Sterility, 17(4): 457-466.

19. Sherman J (1990): Cryopreservation of human semen. In: CRC handbook of the laboratory diagnosis and treatment of infertility. Keel B, Webster B, eds. Boca Raton, CRC Press, Pp. 229-259.

20. Katkov II, Bolyukh VF, Chernetsov OA et al. (2012): Kinetic Vitrification of Spermatozoa of Vertebrates: What Can We Learn from Nature? Current Frontiers in Cryobiology, 3:34-8.

21. Isachenko V, Maettner R, Petrunkina AM et al. (2011): Cryoprotectant free Vitrification of Human Spermatozoa in Large (to $0.5 \mathrm{~mL}$ ) Volume: Novel Technology. Clin Lab., 57:643-650.

22. Punyatanasakchai $P$, Sophonsritsuk A, Weerakiet $S$ et al. (2008): Comparison of cryopreserved human sperm in vapor and liquid phases of liquid nitrogen: effect on motility parameters, morphology, and sperm function. Fertil Steril., 90:1978-82.

23. Moskovtsev SI, Kuznyetsov V, Spiridonov S et al. (2011): Comparison of vitrification and slow vapor protocols for cryopreservation of human spermatozoa. In: Materials of 57th Canadian. Fertility and Andrology Society Annual Meeting. https://cfas.ca > annualmeeting. 